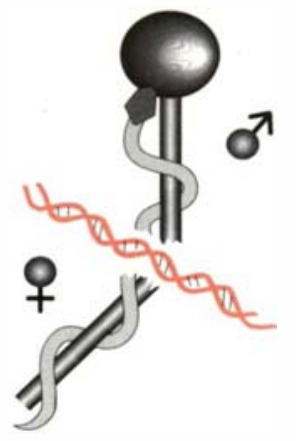

Avis du Comité consultatif national d'éthique pour les sciences de la vie et de la santé (France)
Comité consultatif national d'éthique pour les sciences de la vie et de la santé, 101 , rue de Tolbiac, 75654 Paris Cedex 13, France.

\title{
La coopération dans le domaine de la recherche biomédicale entre équipes françaises et équipes de pays en voie de développement économique
}

Les projets de recherche biomédicale entre les équipes françaises et les équipes de pays en voie de développement économique exigent des précautions particulières afin que soient garanties la dignité et la sécurité des personnes se prêtant à ces recherches d'intérêt général.

1. Un accord de coopération entre les autorités gouvernementales françaises et les pays ou régions impliqués doit préciser les conditions de la formation et de l'encadrement des chercheurs et du personnel sanitaire participant à l'étude projetée.

2. Des études préalables réalisées par une ou plusieurs équipes indépendantes de celles appelées à réaliser le projet doivent permettre d'identifier les particularités sociales, politiques, religieuses ou culturelles susceptibles d'empêcher sa réalisation ou d'en contrarier le bon déroulement.

3. Tous les projets doivent être soumis avant que d'être entrepris à un comité spécialisé à créer (Comité consultatif français de protection des personnes se prêtant à la recherche biomédicale dans les pays en voie de développement ou CCPVD).

Ce comité spécialisé doit notamment inclure des experts de l'Organisation mondiale de la santé ayant l'expérience de la conduite ou du contrôle de tels projets et pourrait être constitué sous l'égide des départements ministériels en charge de la Santé, de la Recherche et de la Coopération. Ce comité pourra sol- liciter l'avis du Comité consultatif national d'éthique français.

4. Les projets seront également et indépendamment étudiés par un Comité d'éthique du pays ou de la région où doit se dérouler l'étude projetée. Une liste de ces Comités d'éthique locaux ou régionaux ainsi que des Comités de défense des droits de l'homme existants doit être établie, publiée et remise à jour annuellement. En l'absence de Comité d'éthique ou des droits de l'homme local ou national, des instances régionales, fonctionnant sous l'égide de l'Organisation mondiale de la santé de l'UNESCO ou du Centre international de l'enfance, pourraient être sollicitées.

Les analyses et avis du Comité spécialisé (CCPPVD) et des Comités locaux nationaux ou régionaux seront soumis aux autorités assurant le financement du projet et autorisant sa mise en œuvre avant qu'il ne soit entrepris.

Le CCNE rappelle dans le présent avis les principes intangibles de la non-commercialisation du corps humain et du consentement.

5. Enfin, les résultats de l'étude feront l'objet d'une publication communiquée aux autorités nationales, aux instances qui sont à l'origine du projet et aux Comités d'éthique qui ont eu à en connaître. Ces résultats doivent être accessibles à la communauté scientifique et au public par voie de publication dans une revue à comité de lecture ou sous toute autre forme. 